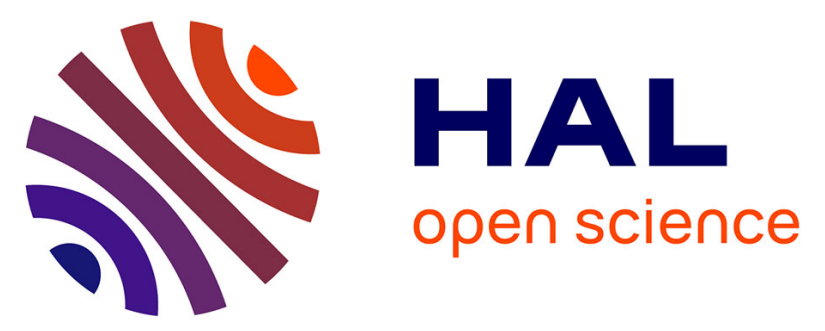

\title{
Temperature dependence of fast fluctuations in single- and double-stranded DNA molecules. A neutron scattering investigation.
}

Elena Cornicchi, Sara Capponi, Matteo Marconi, Giuseppe Onori, Alessandro Paciaroni

\section{To cite this version:}

Elena Cornicchi, Sara Capponi, Matteo Marconi, Giuseppe Onori, Alessandro Paciaroni. Temperature dependence of fast fluctuations in single- and double-stranded DNA molecules. A neutron scattering investigation.. Philosophical Magazine, 2007, 87 (3-5), pp.509-515. 10.1080/14786430600909022 . hal-00513742

\section{HAL Id: hal-00513742 \\ https://hal.science/hal-00513742}

Submitted on 1 Sep 2010

HAL is a multi-disciplinary open access archive for the deposit and dissemination of scientific research documents, whether they are published or not. The documents may come from teaching and research institutions in France or abroad, or from public or private research centers.
L'archive ouverte pluridisciplinaire HAL, est destinée au dépôt et à la diffusion de documents scientifiques de niveau recherche, publiés ou non, émanant des établissements d'enseignement et de recherche français ou étrangers, des laboratoires publics ou privés. 


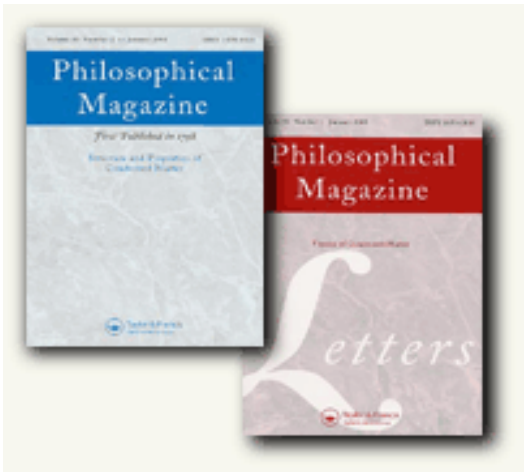

\section{Temperature dependence of fast fluctuations in single- and double-stranded DNA molecules. A neutron scattering investigation.}

\begin{tabular}{|r|l|}
\hline Journal: & Philosophical Magazine \& Philosophical Magazine Letters \\
\hline Manuscript ID: & TPHM-06-May-0153.R1 \\
\hline Journal Selection: & Philosophical Magazine \\
\hline Date Submitted by the & 05-Jul-2006 \\
\hline Complete List of Authors: & $\begin{array}{l}\text { Cornicchi, Elena; University of Perugia, Physics } \\
\text { Capponi, Sara; UPV-EHU, Facultad de Química, , Departamento de } \\
\text { Física de Materiales } \\
\text { Marconi, Matteo; University of Perugia, Physics } \\
\text { Onori, Giuseppe; University of Perugia, Physics } \\
\text { paciaroni, alessandro; University of Perugia, Physics }\end{array}$ \\
\hline Keywords: & biomolecules, neutron scattering \\
\hline Keywords (user supplied): & dynamical transition, DNA \\
\hline
\end{tabular}

\section{(s) ScholarONE" \\ Manuscript Central}




\begin{abstract}
Through elastic neutron scattering measurements we have investigated the picosecond dynamics of dry and hydrated powders of DNA in the double-stranded (dsDNA) and single-stranded (ssDNA) state, in the wide temperature range from 20 to $300 \mathrm{~K}$. The extracted mean square displacements of DNA hydrogen atoms exhibit an onset of anharmonicity at around $100 \mathrm{~K}$. The dynamics of the hydrated samples shows a further anharmonic contribution appearing at a temperature $T_{d}=230-240 \mathrm{~K}$. Such dynamical behaviour is similar to the well-studied dynamical transition found in hydrated protein powders. The mean square displacements of dsDNA and ssDNA are practically superimposed in the whole temperature range for both dry and hydrated samples. This suggests that the DNA local mobility in the picosecond timescale does not depend on the single- or double-stranded conformation.
\end{abstract}




\section{Introduction}

Deoxyribonucleic acid (DNA) is arguably the most important biomolecule due to its role of storage and transmission of all the genetic information regarding structure and function of every living cell. The study of the dynamical aspects of the DNA structure is of great interest, as the main functions exploited by this biomolecule require a great flexibility. Since the beginning of 1960s, experiments using hydrogen-deuterium exchange suggested that even at low temperatures DNA is subject to local structural fluctuations which result in broken interbases hydrogen bonds and in opening and closing of the structure at nucleotide pair levels [1]. These structural slow motions, like those involving bending and twisting of the double helix, allow and control the accessibility of the structure to host molecules such as nucleases and polymerases. Through nuclear magnetic resonance (NMR) studies [2], it has been shown that, inside an intact double helix, the deoxyribose and the sugar-phosphate backbone fluctuate from their equilibrium geometry, and bases undergo libration motions with correlation times of the order of nanoseconds or longer. Electronic spin resonance measurements [3] have revealed the existence of base motions on the nanosecond timescale in RNA and DNA single- and double-stranded systems. In addition, fast conformational motions in the picosecond and nanosecond time-window, already revealed by molecular dynamics (MD) [4,5] and time-resolved fluorescence depolarization [6] measurements, seem to be crucial in many functionally important processes such as chemical reactions involving local interactions with the reacting site and sequences recognition by enzymes [7].

A major role in determining the DNA conformational dynamics is played by hydration water [8], which is important for stabilisation and function as well [9-11]. The influence of water on conformation of nucleic acid has been the subject of many investigations [9,10,12], since also the DNA structure was discovered to be dependent on the relative humidity $[10,13]$. Hydration is also relevant regarding DNA interactions with other biomolecules [14], in particular in DNA-protein interactions. On these grounds, understanding how water affects the nucleic acid dynamics is important from both applied and basic science point of view.

In the picosecond and nanosecond timescale, the dynamical behaviour of hydrated biopolymers is characterized by an onset of stochastic large-amplitude fluctuations taking place in the temperature range 200 - $240 \mathrm{~K}$ [15-21]. In proteins [15-18], DNA [19,20] and tRNA [21], this dynamical transition has been supposed to be ruled by the hydration water, which would increase the structural flexibility of the biomolecule, by allowing the sampling of a large number of spatial conformations. In addition, such a phenomenon has been also interpreted in terms of the Mode Coupling theory [15,19,20].

Here we present the results of a detailed elastic neutron scattering investigation performed to characterise the amplitude of DNA fast motions as a function of temperature. This fast dynamics has been studied for the double-stranded DNA (dsDNA) and unfolded not base stacking single-stranded DNA (ssDNA). To clarify the differences in the dynamics of these two three-dimensional conformations is important since in many biological processes in the cell, such as transcription of genes, DNA double helix separates into singlestranded DNA. We tested, within the accuracy of our measurements, whether the picosecond dynamics is or not dependent on the spatial arrangements of the nucleic acid. 


\section{Materials And Methods}

\subsection{Samples preparation}

Lyophilised DNA from salmon testes in the double-strand state was purchased from Sigma-Aldrich (St. Louis, MO). It was previously dissolved in heavy water (Sigma) by leaving in solution $1 \mathrm{~g}$ of DNA in $20 \mathrm{ml}$ of $\mathrm{D}_{2} \mathrm{O}$ at room temperature for 1 day, to properly substitute all the exchangeable hydrogen atoms with deuterium [1]. Single-stranded DNA has been obtained by thermal denaturation of the native dsDNA. In order to verify that the denaturation process was not reversible, we repeated the DSC measurement on the previously denatured DNA sample. As no peak appeared in the second run, we can conclude that a possible re-association of the single strands during the cooling process can be discarded. Both the solution of ssDNA and dsDNA were freeze-dried and further dehydrated under vacuum in presence of $\mathrm{P}_{2} \mathrm{O}_{5}$ in order to obtain the lowest achievable water content (about a few percent). The samples were subsequently hydrated by $\mathrm{D}_{2} \mathrm{O}$ vapour exchange, putting them in presence of a $\mathrm{KCl}$ saturated solution of $\mathrm{D}_{2} \mathrm{O}$. The hydration level was determined by weighing the sample before and after the hydration process. We obtained the following samples ( $h=$ grams water/grams DNA): ssDNA at $0 h$ and $0.55 h$, dsDNA at $0 h$ and $0.55 h$. The hydration degree of $0.55 \mathrm{~h}$ corresponds approximately to the complete coverage of the first hydration shell of dsDNA [22]. Due to the high incoherent neutron cross section of protons the signal we revealed comes mainly from non-exchangeable DNA hydrogen atoms [23].

\subsection{Incoherent neutron scattering}

Thermal neutrons are powerful and widely used probes to directly obtain information on the fast motions of biological samples. Neutrons exchange with sample nuclei a momentum $\hbar \boldsymbol{Q}$ and an amount of energy $E=\hbar \omega$ with a probability given by the dynamical structure factor $S(Q, E)$. Since the samples are isotropic, the measured dynamical structure factor depends only on the modulus $Q$ of the wave-vector transfer.

In the incoherent approximation the elastic intensity can be described by the following law [16,23]:

$$
S(Q, E \approx 0) \approx e^{-\left\langle u^{2}\right\rangle_{G} Q^{2}} A_{0}(Q)
$$

In this equation, the so-called Debye-Waller factor $e^{-\left\langle u^{2}\right\rangle_{G} Q^{2}}$ takes into account for the $Q$-dependence of the elastic intensity due to the vibrational atomic mean square displacements (MSD) $\left\langle u^{2}\right\rangle_{G}$ of protons in the bottom of their potential wells. The term $A_{0}(Q)$ is the elastic incoherent structure factor (EISF), the $Q-$ dependence of which provides information on the geometry of the investigated motions.

Within the simple framework of the double-well model, already employed to describe the dynamics of proteins [15,16], the hydrogen atoms are considered dynamically equivalent and their motions are schematized as jumps between two distinct sites with a different free energy. The corresponding EISF is:

$$
A_{0}(Q)=1-2 p_{1} p_{2}\left(1-\frac{\sin (Q d)}{Q d}\right)
$$


where $d$ is the mean spatial distance between the two potential wells, and $p_{1}$ and $p_{2}$ are the occupation probabilities of the ground and the excited state respectively. From Eqs. (1)-(2) the total MSD of H atoms can be derived via the following relationship $[15,16]$ :

$$
<u^{2}>_{t o t}=-\left[\frac{d \ln S(Q, E \approx 0)}{d\left(Q^{2}\right)}\right]_{Q=0}=<u^{2}>_{G}+\frac{1}{3} p_{1} p_{2} d^{2}
$$

In this equation, the second term $p_{1} p_{2} d^{2} / 3$ quantifies the proton mobility due to jumping between the two energetic sites.

\subsection{Neutron scattering experiment}

The measurements were performed at the backscattering spectrometer IN13 (ILL, Grenoble). The energy resolution of $4.5 \mu \mathrm{eV}$ (HWHM) allowed us to resolve motions faster than about $150 \mathrm{ps}$. The data were collected in the wide $Q$-range $0.2-4.9 \AA^{-1}$, with an average wave-vector transfer resolution of $\sim 0.2 \AA^{-1}$. The sample holder, a standard flat aluminium cell with inner thickness of $1 \mathrm{~mm}$, was placed at an angle of $120^{\circ}$ with respect to the incident beam direction. All the samples were investigated from 20 to $300 \mathrm{~K}$. The acquired data were corrected in order to take into account for incident flux, cell scattering, self-shielding and detector responses. Then, the elastic intensity of each sample relative to a given temperature was normalized with respect to the data collected at the lowest measured temperature. Since an average transmission of about $90 \%$ was obtained, we neglected the contribution due to multiple scattering processes.

\section{Results and Discussion}

The characteristic $T$ - and $Q$-dependence of the measured elastic intensity of the dsDNA hydrated sample is shown in Fig. 1. In the whole T-range the data are quite well reproduced by using Eq. (1). We found that for temperatures above around $100 \mathrm{~K}$ the elastic intensity exhibits a slight non-Gaussian behaviour. In terms of the double-well model this behaviour corresponds to the onset of anharmonic motions, i.e. jumps between the two sites. As temperature increases, the departure from the Gaussian trend becomes more and more evident. Recently, NMR [24] and neutron scattering [25] studies on proteins found a low-T anharmonic dynamics which was attributed principally to reorientation motions of methyl groups. This idea has been supported by the finding that in wheat germ tRNA powders, characterised by a paucity of methyl groups, such lowtemperature anharmonicity is absent [21]. In salmon DNA approximately $9 \%$ of the non-exchangeable hydrogen atoms belong to $\mathrm{CH}_{3}$ groups, located in thymine nucleobase. On these grounds, we attributed the onset of the anharmonic dynamics above $100 \mathrm{~K}$ to the methyl group motions activation. In this picture, the dynamics is purely vibrational only for temperatures up to approximately $100 \mathrm{~K}$. Therefore, the Gaussian contribution has been approximated with a linear trend as a function of temperature, and the slope was determined from the behaviour of $\left\langle u^{2}(T)\right\rangle_{G}$ vs. $T$ till $100 \mathrm{~K}$. By applying Eq. (1) to fit the measured elastic intensities we found a value of $d=1.1 \pm 0.2 \AA$ nearly constant with $\mathrm{T}$ and hydration degree. This quantity corresponds to the mean spatial length probed by the hydrogen atoms belonging to nucleobases and sugar deoxyribose. Similar values have been reported from neutron scattering measurements on dry and hydrated protein powders $[15,16]$ and attributed to side-chains torsional motions, involving or not methyl groups. 
In Fig. 2 we show the total MSD relative to all the samples, calculated with Eq. (3). At low temperature $\left\langle u^{2}\right\rangle_{t o t}$ display a common linear dependence, with the main contribution provided by the purely vibrational harmonic MSD $\left\langle u^{2}\right\rangle_{G}$. Above $\sim 100 \mathrm{~K}$ a first onset of anharmonicity starts to be slightly visible in all the measured systems. Such dynamics, which becomes more evident with increasing temperature, is consistent with methyl group reorientation, as previously discussed. Then a second anharmonic onset, the so-called dynamical transition, observed only in the hydrated samples, occurs at the estimated temperature $T_{d}=230-$ $240 \mathrm{~K}$. This value turns out to be in agreement with previous theoretical [26] and experimental [19,20,27] results. MD simulations on hexamer oligonucleotide duplex in aqueous solution localised a glass-like dynamical transition in the $223-234 \mathrm{~K}$ range [26]. From dielectric relaxation spectroscopy on hydrated solid calf thymus DNA the transition was found to take place at around $238 \mathrm{~K}$ [27], while through a quasielastic neutron scattering study on Li-DNA fibres Sokolov and co-workers [19,20] found the rise of a strong anharmonicity above $210 \mathrm{~K}$ for samples hydrated at $11 \%$ or more. In this work, the authors suggested that the hydration shell plays a crucial role in the DNA dynamics [19] and the origin of the glass transition was interpreted as the melting of hydrogen bonds of bound water molecules on the surface of DNA, in analogy with protein powders $[16,28]$. The fact that the dynamical transition is not revealed in the dry samples (see Fig. 2) supports such a picture. Quite remarkably, the total MSD of ss- and dsDNA are practically coincident, within our range of accuracy, thus suggesting that the picosecond dynamics described in this study does not depend on the three-dimensional conformation of the nucleic acid. The temperature response of DNA is the same, irrespective of the single- or double-strand spatial structure. DSC studies [22] showed that the transformation from double-stranded helix to single-stranded chains was found to be accompanied by melting of the structural part of the hydration shell of dsDNA. These water molecules, which constitute the so-called dynamically ordered water, are mainly located in the minor and major grooves of the duplex structure [29]. Our findings suggest that the lack of such water molecules in ssDNA does not influence the internal dynamics of the nucleic acid in the time-window accessible in the present experiment.

\section{Acknowledgments}

The authors are grateful to ILL (Institut Laue-Langevin, Grenoble, France) and to the Italian-French CRG IN13 for providing beam time at the IN13 spectrometer.

\section{References}

[1] M. P. Printz and P. H. von Hippel, Proc. Natl. Acad. Sci. USA 53363 (1965) and references therein.

[2] M. E. Hogan and O. Jardetzky, Proc. Natl. Acad. Sci. USA 766341 (1979).

[3] S.-C. Kao and A. M. Bobst, Biochemistry 245465 (1985).

[4] M. A. Young et al., J. Am. Chem. Soc. 11959 (1997).

[5] L. Yang and B. M. Pettitt, J. Phys. Chem. 1002564 (1996).

[6] D. P. Millar et al., Proc. Natl. Acad. Sci. U.S.A. 775593 (1980).

[7] E. B. Brauns et al., J. Am. Chem. Soc. 1202449 (1998). 
[8] A. M. Ababneh et al., Biophys. J. 851111 (2003).

[9] S. K. Pal et al., Proc. Natl. Acad. Sci. USA 1008113 (2003).

[10] G. Albiser et al., Int. J. Biol. Macromolecules 28199 (2001).

[11] I. A. Beta et al., Chem. Phys 292451 (2002).

[12] S. Rüdisser et al., J. Phys. Chem. 100458 (1996).

[13] R. E. Franklin and R. G. Gosling, Acta Crystallogr. 6673 (1953).

[14] M. C. R. Symons, Cell. Mol. Life Sci. 57999 (2000).

[15] W. Doster et al., Nature 337754 (1989).

[16] A. Paciaroni et al., Chem. Phys. Lett. 410400 (2005).

[17] E. Cornicchi et al., Phys. Rev. Lett. 95158104 (2005).

[18] E. Cornicchi et al., Biophys. J. 91289 (2006).

[19] A. P. Sokolov et 1., J. Chem. Phys. 1107053 (1999).

[20] A. P. Sokolov et al., J. Biol. Phys. 27313 (2001).

[21] G. Caliskan et al., J. Am. Chem. Soc. 12832 (2006).

[22] G. M. Mrevlishvili et al., Thermochimica Acta 39473 (2002).

[23] M. Bée, Quasielastic Neutron Scattering (Adam Hilger, Bristol and Philadelphia) (1988).

[24] A. L. Lee and A. J. Wand, Nature 411501 (2001).

[25] J. H. Roh et al., Phys. Rev. Lett. 95038101 (2005).

[26] J. Norberg and L. Nilsson, Proc. Natl. Acad. Sci. USA 9310173 (1996).

[27] M. Neubert et al., Proc. Int. Symp. Electrets, 5th, 825 (1985).

[28] W. Doster and M. Settles, Biochim. Biophys. Acta 1749173 (2005).

[29] S. K. Pal et al., Proc. Natl. Acad. Sci. USA 10013746 (2003). 


\section{Figure captions}

Figure 1. Elastic neutron scattering intensities vs. $Q^{2}$ for the hydrated dsDNA at T =50,100, 160, 220, 280K, from top to bottom. Lines correspond to fitting with Eq. (1).

Figure 2. Temperature dependence of the total mean square displacements for ssDNA at $0 h$ (empty squares), ssDNA at $0.55 h$ (empty circles), dsDNA at $0 h$ (full squares) and dsDNA at $0.55 h$ (full circles). The dashed line is the Gaussian contribution $\left\langle u^{2}\right\rangle_{G}$ [see Eq. (3)] to the total MSD of dsDNA at $0 h$. 


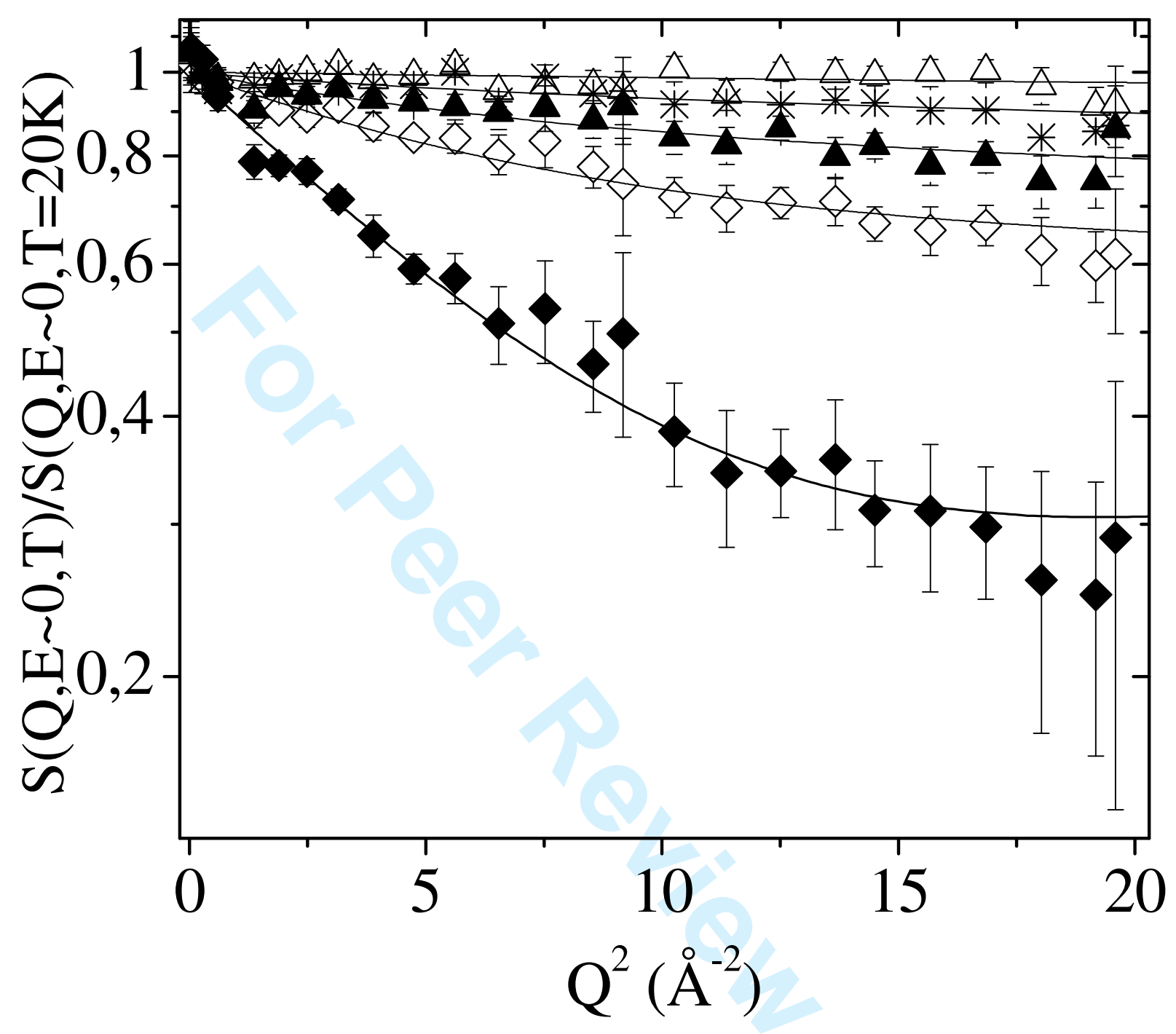

Figure 1. 


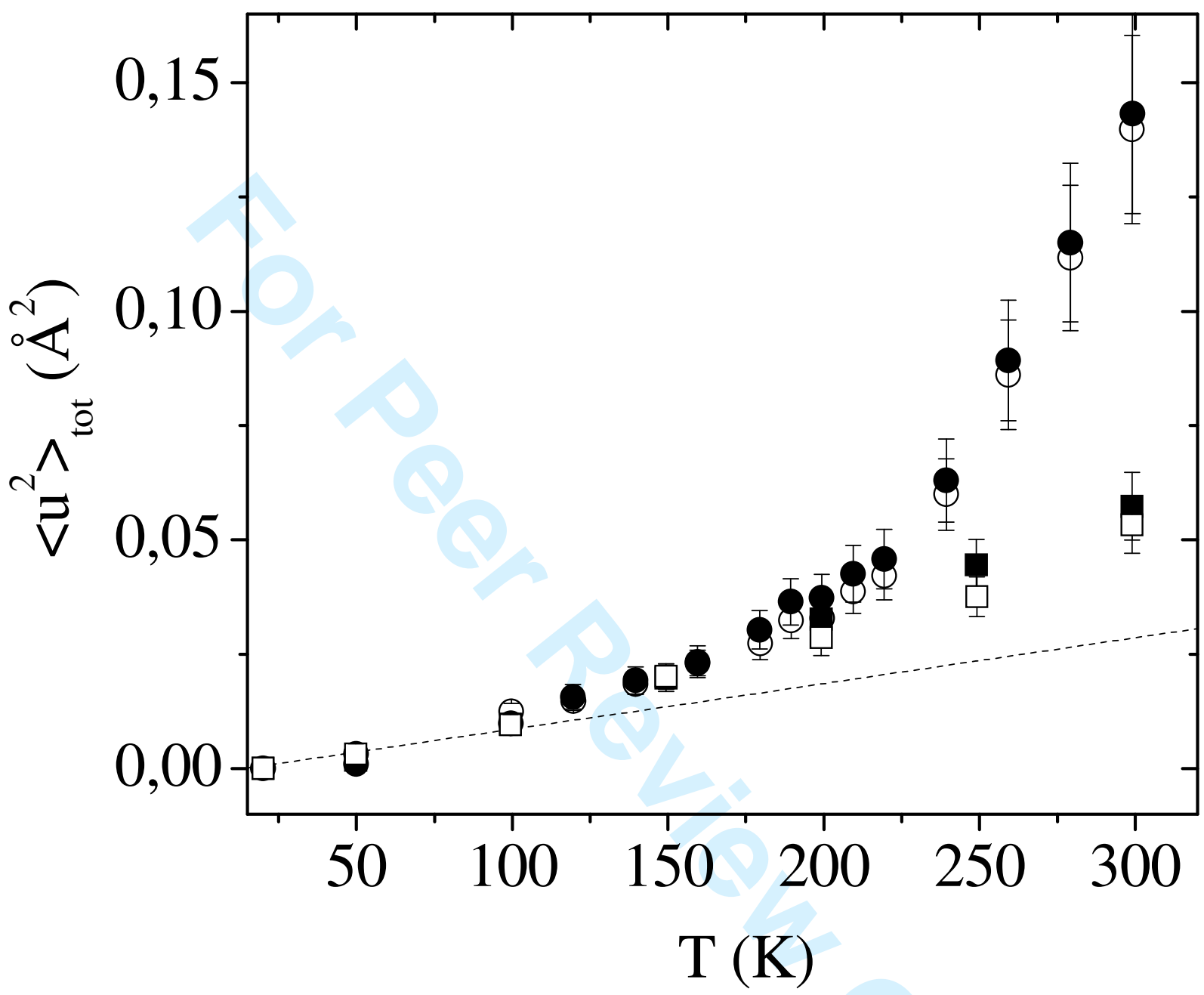

Figure 2. 